\title{
The importance of feeding status and desiccation rate in successful anhydrobiosis of Panagrolaimus detritophagus
}

\author{
Shakiba SAlEHIAN ${ }^{1}$, Bart P. BRAECKMAN ${ }^{2}$, Lynda BELADJAL ${ }^{1}$, Wim BerT ${ }^{3}$, \\ James S. CLEGG ${ }^{4}$ and Johan MERTENS ${ }^{1, *}$ \\ ${ }^{1}$ Terrestrial Ecology Unit, Biology Department, Ghent University, K.L. Ledeganckstraat 35, \\ B-9000 Ghent, Belgium \\ ${ }^{2}$ Research Group for Aging Physiology and Molecular Evolution, Biology Department, \\ Ghent University, K.L. Ledeganckstraat 35, B-9000 Ghent, Belgium \\ ${ }^{3}$ Nematology Section, Biology Department, Ghent University, K.L. Ledeganckstraat 35, \\ B-9000 Ghent, Belgium \\ ${ }^{4}$ Bodega Marine Laboratory and Section of Molecular \& Cellular Biology, University of California, \\ Davis, Bodega Bay, CA 94923, USA
}

Received: 3 February 2010; revised: 10 May 2010 Accepted for publication: 10 May 2010

\begin{abstract}
Summary - We examined the effect of nutritional status and desiccation rate on the ability of Panagrolaimus detritophagus to undergo anhydrobiosis, as well as to survive high temperatures in the dried state. Both nutrition and drying rate were found to be important, with starvation and slow drying providing better success at anhydrobiosis. The upper temperature for survival of dried animals in laboratory studies was $80^{\circ} \mathrm{C}$. Starved worms recovered from drying more successfully when the starvation period was followed by a smooth, gradual dry period prior to undergoing desiccation. Thus, the ability of these worms to enter and leave anhydrobiosis is dependent on critical stress signals.
\end{abstract}

Keywords - desiccation, heat resistance, nematodes, starvation.

Organisms that tolerate complete dehydration are said to be anhydrobiotic and can be referred to as anhydrobiotes. This remarkable ability exists across all biological kingdoms. In animals, anhydrobiosis occurs in nematodes, rotifers, tardigrades and some arthropods (Alpert, 2005a). These taxa, except for nematodes, not only tolerate desiccation, but also survive high temperatures, some well above $100^{\circ} \mathrm{C}$ (Mertens et al., 2008).

Temperature and humidity are the most critical factors determining nematode distributions in nature (Bakonyi et $a l ., 2007)$. Free-living nematodes are ubiquitous inhabitants of soils in most ecosystems (Treonis \& Wall, 2005), ranging from the Antarctic dry valleys to hot deserts and agro-ecosystems (Treonis et al., 2000; Wharton, 2002, 2003; Brown et al., 2004; Shannon et al., 2005). Most nematodes are small and lack a rigid skeleton of any kind, features that enable them to shrink and curl as they dry out. Such morphological changes allow nema-

*Corresponding author, e-mail: Johan.Mertens@UGent.be todes to reduce their surface area, thereby lessening water loss (Alpert, 2006). Due to their small size and inability to undergo long distance migration, free-living nematodes must react quickly when desiccation stress begins (McSorley, 2003). Many species enter anhydrobiosis when facing desiccation (Wharton, 1996; Ricci \& Pagani, 1997; Treonis et al., 2000; McSorley, 2003; Brown et al., 2004; Alpert, 2005a, b, 2006; Shannon et al., 2005; Treonis \& Wall, 2005).

At high temperatures metabolism is likely to be reduced because nematodes become quiescent and first exhibit heat stupor, and then heat coma. Recovery after exposure to heat coma has been reported in a few studies (Wharton, 2002). In this study we explored the extent of flexibility when entering and subsequently recovering from anhydrobiosis in Panagrolaimus detritophagus Fuchs, 1930, both in relation to the nutrititional status and desiccation 
rate of the worms. We also examined their survival at high temperatures with regard to these parameters.

\section{Materials and methods}

\section{MorphologicAl AND MOLECUlar CHARACTERISATION}

Nematode specimens for light microscopy observations were collected using a stereomicroscope (Wild M5), placed in an embryo dish, and fixed in hot aqueous $4 \%$ formaldehyde $+1 \%$ glycerin and processed to anhydrous glycerin following the glycerin-ethanol method (De Grisse, 1969). DNA extraction, PCR reaction and sequencing the SSU (18S) rDNA was done as in Bert et al. (2008). For the D2D3 region, the forward D2A (5'ACAAGTACCGTGAGGGAAAGTTG-3') and reverse D3B (5'-TCCTCGGAAGGAACCAGCTACTA-3') primers were used (Koekemoer et al., 1999).

Nearly complete sequences of $18 \mathrm{~S}$ rRNA (1685 bp) and the D2D3 expansion region of 28S rRNA (727 bp) were obtained. The two sequences were deposited in NCBI GenBank under accession numbers GU014546 and GU014547.

Additional sequences of Panagrolaimus for the phylogenetic analyses were obtained from GenBank, mainly from Lewis et al. (2009) and Shannon et al. (2005). The sequences were aligned with Clustal W (Thompson et al., 1994) and manually checked. Bayesian Inference (BI) was performed with MrBayes v3.1.2 (Ronquist \& Huelsenbeck, 2003) with a general time-reversible model with rate variation across sites and a proportion of invariable sites (GTR $+\mathrm{I}+\Gamma$ ), as estimated by PAUP*/Mr Modeltest 1.0b (Nylander, 2004). Analyses were run for $3 \times 10^{6}$ generations and trees were generated using the last $2 \times 10^{6}$ generations well beyond the burn-in value.

\section{CULTURing}

Stems of Lobularia maritima plants (Brassicaceae) were collected in the Canary Islands, Province of Santa Cruz de Tenerife (Adeje, Barranco del Infierno $28^{\circ} 7^{\prime} 21^{\prime \prime} \mathrm{N}$, $16^{\circ} 43^{\prime} 3^{\prime \prime} \mathrm{W}$ ), air-dried and stored for collection of seeds. These seeds were rehydrated for germination on nutrient agar in Petri dishes. Among the grains that did not germinate, an expanding population of Panagrolaimus flourished, and this was used for the inoculation of our laboratory culture.

Panagrolaimus are bacterial-feeding soil nematodes and can be cultured in the laboratory using the meth- ods developed for Caenorhabditis elegans (Shannon et al., 2005). The worms were cultured at $20^{\circ} \mathrm{C}$ on Petri dishes containing nutrient agar (Oxoid CM0003; Oxoid, Basingstoke, UK) with a lawn of Escherichia coli K12 as a food source. In the starvation experiments, part of the worm population was placed on plates containing agar $\mathrm{N}^{\circ} 1$ (Oxoid LP0011) without E. coli.

\section{DESICCATION}

Desiccation tolerance was monitored by taking $20 \mu \mathrm{l}$ of a mixed stage culture that was rinsed off the plate with $\mathrm{S}$ buffer $(0.1 \mathrm{M} \mathrm{NaCl}, 0.05 \mathrm{M}$ potassium phosphate buffer, $\mathrm{pH}$ 6.0). In the starvation-re-feeding experiment, $20 \mu \mathrm{l}$ aliquots (about 50 nematodes), were put on glass slides and allowed to dry at room temperature (65\% humidity) prior to being transferred to a desiccator over silica gel. The dried samples were kept in the desiccator for 2 days.

In the slow dehydration experiment, the glass slides containing the $20 \mu \mathrm{l}$ aliquots of mixed-stage worm suspension were transferred to a series of sealed boxes with decreasing relative humidity $(\mathrm{RH})$ (Winston \& Bates, 1960). The worms remained in each box for $2 \mathrm{~h}$ before transfer to the next box with lower relative humidity. In the final stage, the worms with $20 \% \mathrm{RH}$ were transferred to a desiccator. The humidity in the boxes was obtained using the following saturated salt solutions, all at $20^{\circ} \mathrm{C}$ :

1. $\mathrm{H}_{2} \mathrm{O}: 100 \% \mathrm{RH}$

2. $\mathrm{NaClO}_{3}: 75 \% \mathrm{RH}$

3. $\mathrm{Na}_{2} \mathrm{HSO}_{4}: 52 \% \mathrm{RH}$

4. $\mathrm{KC}_{2} \mathrm{H}_{3} \mathrm{O}_{2}: 20 \% \mathrm{RH}$

5. Entering into desiccator: $0 \% \mathrm{RH}$

To have a stabilised vapour pressure in each box, the solutions were prepared a few days in advance, and RH was monitored (Testo 635; Testo, Alton, UK). All samples dried over silica gel were rehydrated by adding distilled water, and survival (scored as movement of the worms after $6 \mathrm{~h}$ of recovery) was assessed under a stereomicroscope (Wild M5). Metabolism and water contents increased rapidly when anhydrobiotic nematodes were immersed in water (Barrett, 1982; Wharton et al., 1985), but there was a period of apparent inactivity referred to as the 'lag phase' which lasts 2-3 h before complete activity resumes (Wharton et al., 1985).

\section{RESISTANCE TO HIGH TEMPERATURES}

The methodology used for heating was the same as that used for the slow heating experiments described by 
Mertens et al. (2008). Heating of dehydrated worms in the dried agar samples was carried out in small aluminium holders put in a dry air oven (Memmert UFE 500; Memmert, Buechenbach, Germany). The probe of a wire thermocouple (connected to a data logger, Testo 175-T3) was placed directly in the sample holder in contact with the dried nematodes. This made us confident that we were measuring the actual temperature of our samples. The specimens reached the desired temperature after $15 \mathrm{~min}$.

\section{Results}

According to light microscopy observations, the few diagnostic morphological/morphometrical characters available in Panagrolaimus point to the species P. detritophagus: relatively small (about $600 \mu \mathrm{m}$ ); lip region simple with rounded lips; clear dorsal denticle at stegostom; and tail conical but asymmetrical, about three anal body diam. long, shorter on ventral side. Phylogenetic analysis based on nearly complete SSU rDNA sequences placed our P. detritophagus sister to P. detritophagus EU543176 (Fig. 1) and only two out of 1685 nucleotides were different. Both populations formed with Panagrolaimus sp. U81579 and Panagrolaimus davidi AJ567385 a highly supported clade (PP: 98). Phylogenetic analysis based on 35 short SSU rDNA sequences to include additional Panagrolaimus populations, of which several were the subject of physiological studies (Shannon et al., 2005; Lewis et al., 2009), resulted in a tree topology with a relatively low resolution (tree topology not shown). This is most likely because of the limited length of the available GenBank sequences. Also our phylogenetic analyses based on the D2D3 region resulted in a tree with low resolution; $P$. detritophagus was placed in a unresolved clade with several Panagrolaimus spp. (result not given).

When a small population of mixed-stage Panagrolaimus is transferred to a fresh agar plate with a lawn of $E$. coli, it will quickly increase in number during the next few days. As the population grows, the food source will be depleted. After several days, the worms will stop proliferating and, finally, development of juveniles will also cease.

We have tested the ability of worms to undergo successful entry into and exit from anhydrobiosis during the course of population expansion, starvation and developmental arrest. Panagrolaimus that went into anhydrobiosis successfully showed typical curled morphology during desiccation time over silica gel, in contrast to that part of the population that did not survive desiccation, having a deformed, stretched shape. This comparison between successful and unsuccessful nematodes was monitored under the stereomicroscope (Wild M5) in both desiccation and rehydration conditions. Worms that curled during desiccation became active after rehydration.

Worms that were put on fresh plates and able to feed ad libitum lacked the ability to enter anhydrobiosis after a normal desiccation procedure (Fig. 2). However, over the course of time, the rate of successful anhydrobiosis rose slowly and reached a maximum of about $50 \%$ in an 11-day-old culture. At that time the plates were already cleared of bacteria. After day 11, the fraction of desiccated surviving worms slowly declined towards a seemingly steady $15-20 \%$ by day 18 .

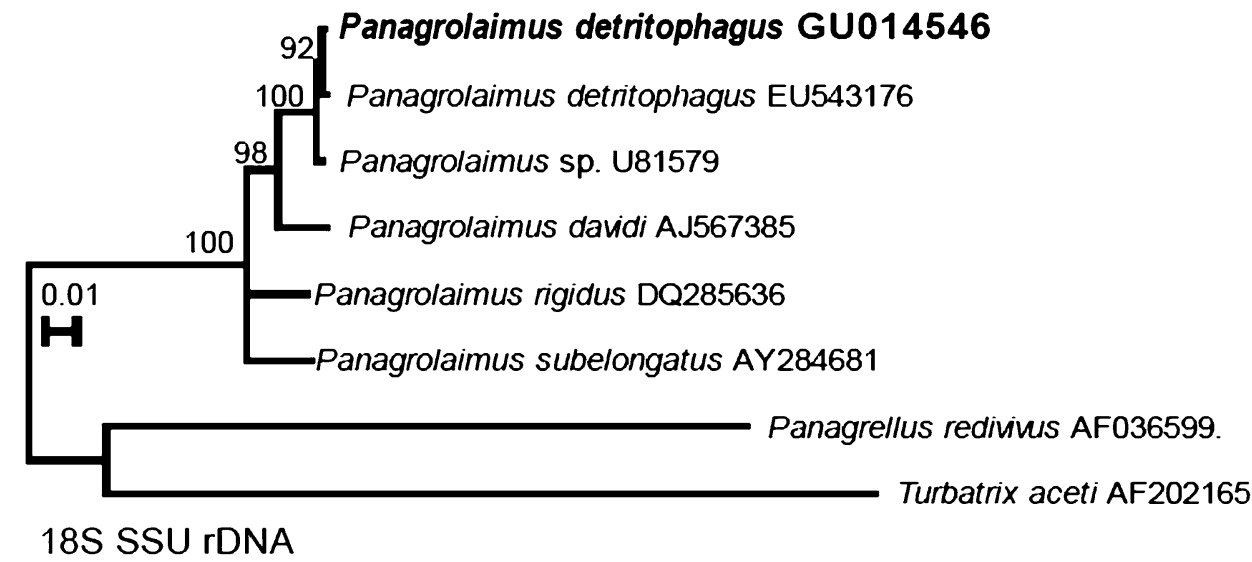

Fig. 1. BI 50\% majority rule consensus phylogeny of Panagrolaimus detritophagus with related sequences from GenBank based on $18 S$ SSU rDNA data. Analysed with a GTR $+I+\Gamma$ model, as estimated by PAUP*/Mr Modeltest 1.Ob. Branch support values are indicated with $P P$ 


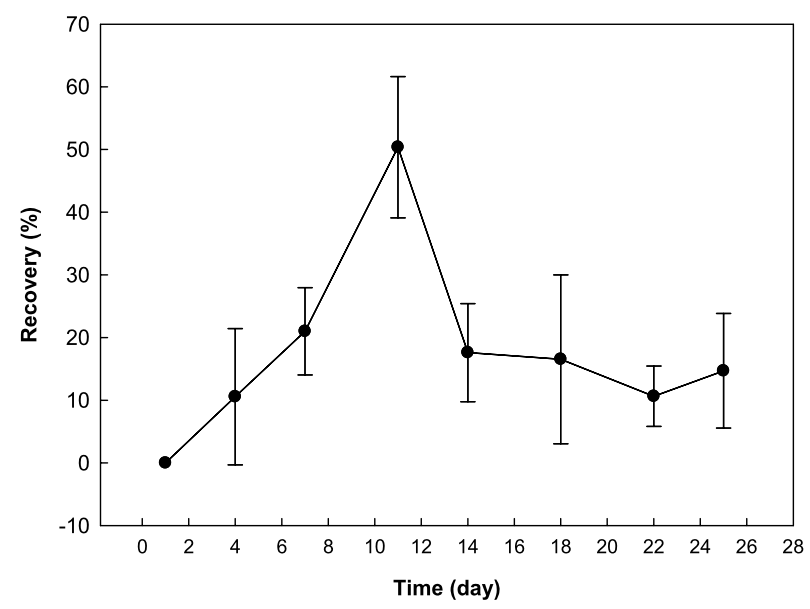

Fig. 2. Desiccation experiment. Mean survival (\%) of Panagrolaimus detritophagus with decreasing food availability in function of time (day). The day in which nematodes were transferred to the fresh palates was referred as day 0. Error bars $=$ standard deviation $(n=7)$.

The intriguing biphasic pattern seen in these results may have been caused by parameters other than feeding conditions (such as population distribution, or humidity/water activity of the agar plates). This prompted us to examine anhydrobiotic success as a function of food availability.

In the re-feeding experiment, nutrient agar plates with lawns of E. coli were seeded with mixed Panagrolaimus populations and the fraction of survival after anhydrobiosis was determined over time in a set of subsamples. After animals on plates were starved (day 7), they were transferred to different types of fresh plates. One subpopulation was transferred to fresh nutrient agar plates seeded with $E$. coli (re-feeding); another subpopulation was transferred to empty agar no. 1 plates (complete starvation) and finally, the control population was left on the original plates (Fig. 3).

As expected, anhydrobiotic success continued to rise in the control population, confirming the pattern found in the first experiment. Worms that were switched to empty agar no. 1 plates and suffered from continued starvation showed a very similar pattern, and anhydrobiotic success remained high even over extended periods of starvation. The subpopulation of worms that were re-fed failed to survive desiccation. Only after a few days, when animals were again starved, did the fraction of anhydrobiotic survivors increase. These data suggest that the capacity to recover mainly depended on starvation rather than population distribution or water content on the plates.

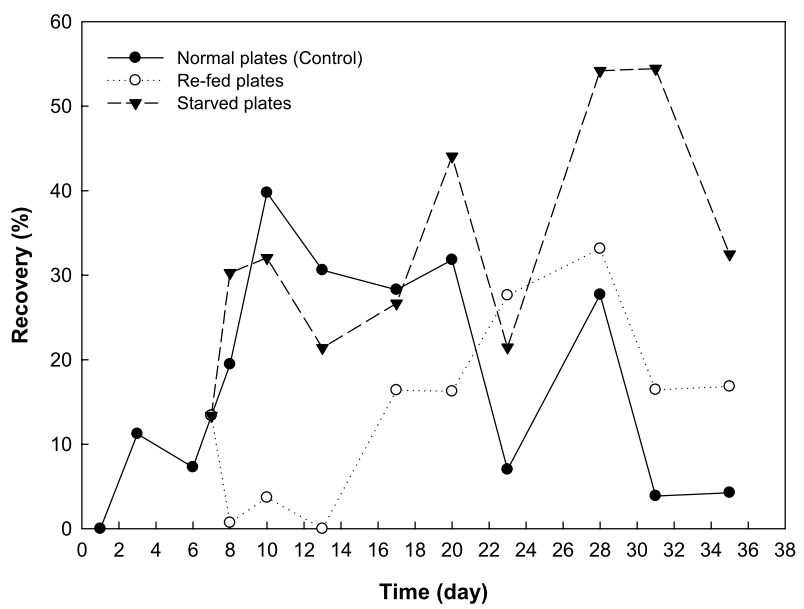

Fig. 3. Starvation-re-feeding experiment. Dynamics of refeeding pattern and survival strategy in Panagrolaimus detritophagus after a complete starvation period in function of time (day) $(n=7)$.

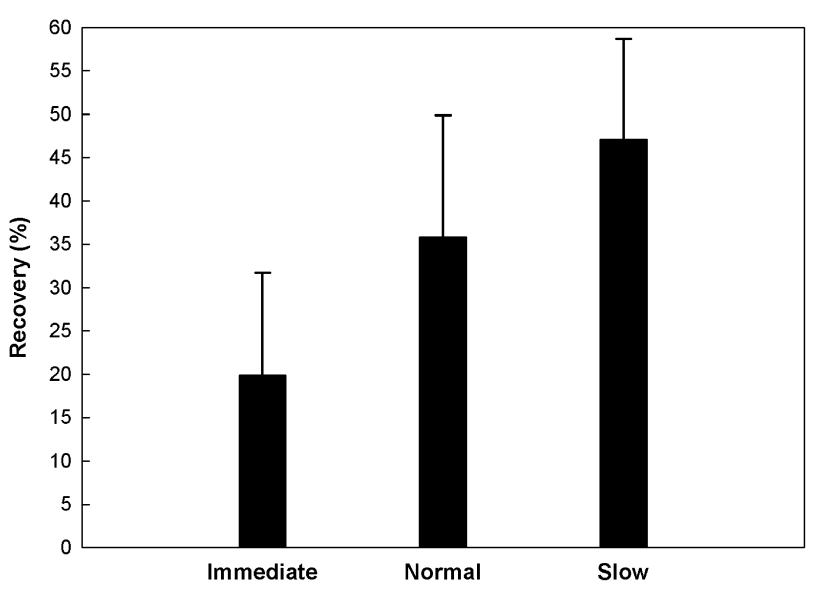

Fig. 4. Recovery (\%) using different rates of desiccation in Panagrolaimus detritophagus $(n=6)$.

We tested the capacity for desiccation recovery under varying rates of desiccation in Panagrolaimus (Fig. 4). Worms were desiccated rapidly $( \pm 3 \mathrm{~h}$, by transferring the $20 \mu \mathrm{l}$ suspensions to a desiccator; referred to as 'Immediate' in Fig. 4), normal ( $\pm 5 \mathrm{~h}$, by allowing $20 \mu \mathrm{l}$ drops to dry at room temperature and humidity) and slow ( $48 \mathrm{~h}$, by controlled decrease in humidity as described in Materials and methods). These worms were in the stage that yielded the highest recovery (day 11; Figs 2, 3 ) in our early experiment. Slow dehydration consistently yielded the highest rates of survival after those worms were rehydrated. This is in accordance with an earlier study reporting increased dehydration survival in several 


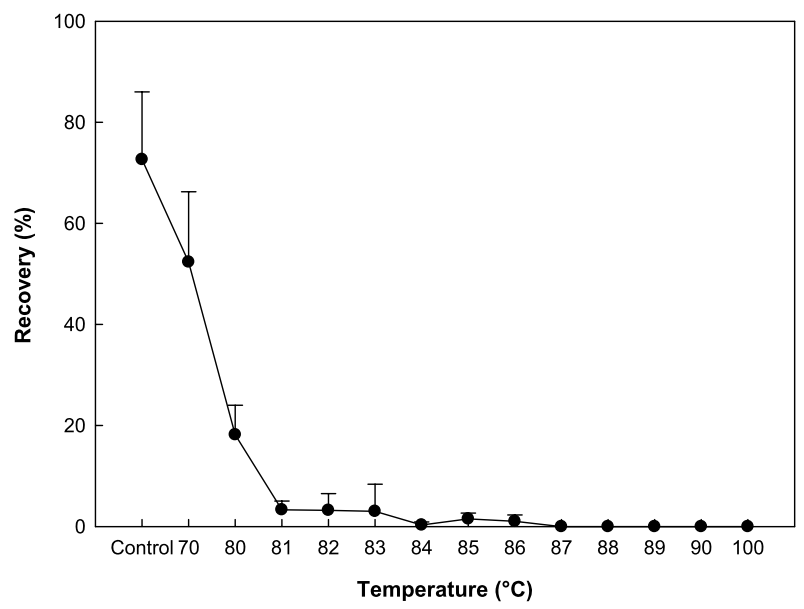

Fig. 5. Heat resistance (mean recovery percentage) of anhydrobiotic Panagrolaimus detritophagus as a function of temperature $\left({ }^{\circ} \mathrm{C}\right)$. Heating of dehydrated worms in the dried agar samples was carried out in small aluminium holders, put in a dry air oven. The probe of a wire thermocouple was placed directly in the sample holder in contact with the dried nematodes. Error bars $=$ standard deviations $(n=8)$.

Panagrolaimus species after a period of preconditioning at $98 \%$ RH (Shannon et al., 2005).

During periods of drought, temperatures can peak to high levels in the natural environment. So we tested the heat resistance of anhydrobiotic Panagrolaimus. Worms were slowly desiccated, and then heated to 70,80, 90 and $100^{\circ} \mathrm{C}$ (Fig. 5). The samples were then cooled to room temperature $\left(20 \pm 2^{\circ} \mathrm{C}\right)$, and the worms were rehydrated as described in Materials and methods and assayed for survival. Refined heating intervals of $1{ }^{\circ} \mathrm{C}$ were used between 80 and $90^{\circ} \mathrm{C}\left(81,82, \ldots, 90^{\circ} \mathrm{C}\right)$. Successful exit from anhydrobiosis was not drastically lowered when worms were exposed to $70^{\circ} \mathrm{C}$. Treatment at $80^{\circ} \mathrm{C}$ showed a prominent decline in survival until $86^{\circ} \mathrm{C}$; while at $90^{\circ} \mathrm{C}$ no anhydrobiotic worms survived (Fig. 5).

\section{Discussion}

Although our paper does not deal with biochemical mechanisms it is obvious that these are important to successful anhydrobiosis. For example, the disaccharide trehalose has long been known to play an important role in the desiccation tolerance of some nematodes (Madin et al., 1978; Behm, 1997) and an interest in the role of trehalose continues for anhydrobiotes in general (Clegg, 2001). In recent years, a lot of attention has been given to the late embryogenesis abundant (LEA) proteins, thought to play a protective role during the anhydrobiosis of a variety of invertebrates (Goyal et al., 2005a; Tunnacliffe \& Wise, 2007) including nematodes (Goyal et al., 2005b). Some information about the genetics of anhydrobiosis in nematodes is also available (Browne et al., 2004; Grewal et al., 2006).

Anhydrobiotic nematodes have been divided into two broad groups: slow-dehydration strategists and fast dehydration strategists (Womersley, 1987). However, it is more likely that a distribution of optimal rates of water loss exists for different species rather than two distinct groups (Allan \& Wharton, 1990). Free-living nematodes are predominantly slow dehydration strategists, surviving the rates of water loss likely to be experienced in their habitats. The variation in survival between different nematode populations seems to be due more to the rates of water loss they can tolerate rather than the absolute degree of desiccation stress they can survive.

Entry into anhydrobiosis is dependent on the activation of a specific genetic programme leading to morphological, behavioural and physiological changes that prepare the worm for desiccation (Ricci \& Pagani, 1997). The execution of this programme and the synthesis of protective metabolites such as trehalose (Behm, 1997) takes several hours at least, which may explain why rapid desiccation is lethal to many anhydrobiotes (Shannon et al., 2005). Compared with other anhydrobiotic species (Mertens et al., 2008), Panagrolaimus is not extremely heat resistant during anhydrobiosis. Remarkably, Shannon et al. (2005) found that the anhydrobiotic potential of Panagrolaimus species and strains is correlated with their phylogenetic position within the genus Panagrolaimus. Our phylogenetic outcome, based on nearly complete $18 \mathrm{~S}$ sequences, is largely similar to that of Shannon et al. (2005): $P$. rigidus AF40 has a sister relation to the clade containing $P$. detritophagus and $P$. davidi, while the clades containing the latter two species also have a sister relation. In Shannon et al. (2005) the strongly anhydrobiotic strains of Panagrolaimus form a single phylogenetic lineage, which is separate from the poorly desiccation-tolerant strains, including $P$. detritophagus. Panagrolaimus detritophagus is known to have a limited ability to survive rapid desiccation but its anhydrobiotic ability improves upon preconditioning (Shannon et al., 2005).

Our results suggest that the ability of $P$. detritophagus to enter into anhydrobiosis is dependent on critical stress signals. Well-fed worms did not survive anhydrobiosis and needed at least a short but vital period of starvation before successfully entering into anhydrobiosis. Although 
starvation was necessary for surviving desiccation and successful rehydration, its duration was critical. Many starved worms that do not have any internal fat reserves for long periods (more than 11 days) were unable to enter anhydrobiosis successfully. Starved worms will recover from drought more successfully if the period of starvation is followed by a smooth gradual drying period prior to undergoing desiccation. Fast dehydration strategists may have acquired adaptations that ensure the necessary slow rate of water loss (Wharton et al., 2008). The latter can be achieved via behavioural mechanisms, such as coiling, which lessens the rate of water loss by reducing the surface area exposed to the air. Coiling is a widespread response to desiccation in nematodes, and appears to be essential for survival (Womersley \& Ching, 1989) in environmental temperatures between $80^{\circ}$ and $90^{\circ} \mathrm{C}$.

\section{References}

Allan, G.S. \& Wharton, D.A. (1990). Anhydrobiosis in the infective juveniles of Trichostrongylus colubriformis (Nematoda: Trichostrongylidae). International Journal for Parasito$\log y$ 20, 183-192.

AlPERT, P. (2005a). The limits and frontiers of desiccationtolerant life. Integrative and Comparative Biology 45, 685696.

AlPERT, P. (2005b). Sharing the secrets of life without water. Integrative and Comparative Biology 45, 683-685.

Alpert, P. (2006). Constraints of tolerance: why are desiccation-tolerant organisms so small or rare? Journal of Experimental Biology 209, 1575-1584.

Bakonyi, G., Nagy, P., Kovacs-Lang, E., Kovacs, E., Barabas, S., Repasi, V. \& Seres, A. (2007). Soil nematode community structure as affected by temperature and moisture in a temperate semiarid shrubland. Applied Soil Ecology 37, 31-40.

BARRETT, J. (1982). Metabolic responses to anabiosis in the fourth stage juveniles of Ditylenchus dipsaci (Nematoda). Proceedings of the Royal Society of London Series B Biological Sciences 216, 159-177.

BEHM, C.A. (1997). The role of trehalose in the physiology of nematodes. International Journal for Parasitology 27, 215229.

Bert, W., Leliaert, F., Vierstraete, A.R., VAnfleteren, J.R. \& Borgonie, G. (2008). Molecular phylogeny of the Tylenchina and evolution of the female gonoduct (Nematoda: Rhabditida). Molecular Phylogenetics and Evolution 48, 728744.

Brown, I.M., Wharton, D.A. \& Millar, R.B. (2004). The influence of temperature on the life history of the Antarctic nematode Panagrolaimus davidi. Nematology 6, 883-890.
Browne, J.A., Dolan, K.M., Tyson, T., Goyal, K., TunNAClifFe, A. \& BURnell, A.M. (2004). Dehydrationspecific induction of hydrophilic protein genes in the anhydrobiotic nematode Aphelenchus avenae. Eukaryotic Cell 3, 966-975.

CLEGG, J.S. (2001). Cryptobiosis - a peculiar state of biological organization. Comparative Biochemistry and Physiology Part B 128, 613-624.

DE GRISSE, A.T. (1969). Redescription ou modification de quelques techniques utilisées dans l'étude des nématodes phytoparasitaires. Mededelingen Rijksfakulteit Landbouwwetenschappen Gent 34, 351-369.

Goyal, K., Walton, L.J., Browne, J.A., Burnell, A.M. \& TUNNACLIFFE, A. (2005a). Molecular anhydrobiology: identifying molecules implicated in invertebrate anhydrobiosis. Integrative and Comparative Biology 45, 702-709.

Goyal, K., Pinelli, C., Maslen, S.L., Rastogi, R.K., STEPhens, E. \& TUNNACLIFFE, A.T. (2005b). Dehydrationregulated processing of late embryogenesis abundant protein in a desiccation-tolerant nematode. FEBS Letters 579, 40934098.

Grewal, P.S., Bornstein-Forst, S., Burnell, A.M., GlaZer, I. \& JAGDALE, G.B. (2006). Physiological, genetic and molecular mechanisms of chemoreception, thermobiosis and anhydrobiosis in entomopathogenic nematodes. Biological Control 38, 54-65.

Koekemoer, L.L., Lochouarn, L., Hunt, R.H. \& CoetZEE, H. (1999). Single-strand conformation polymorphism analysis for identification of four members of the Anopheles funestus (Diptera: Culicidae) group. Journal of Medical Entomology 36, 125-130.

Lewis, S.C., Dyal, L.A., Hilburn, C.F., Weitz, S., Liau, W.S., Lamunyon, C.W. \& Denver, D.R. (2009). Molecular evolution in Panagrolaimus nematodes: origins of parthenogenesis, hermaphroditism and the Antarctic species P. davidi. BMC Evolutionary Biology 9, 15.

Madin, K.A.C., Crowe, J.H. \& Loomis, S.H. (1978). Metabolic transitions in a nematode during induction and recovery from anhydrobiosis. In: Crowe, J.H. \& Clegg, J.S. (Eds). Dry biological systems. New York, NY, USA, Academic Press, pp. 155-184.

MCSORLEY, R. (2003). Adaptations of nematodes to environmental extremes. Florida Entomologist 86, 138-142.

Mertens, J., Beladjal, L., Alcantara, A., Fougnies, L., VAN Der Straeten, D. \& CLEGG, J.S. (2008). Survival of dried eukaryotes (anhydrobiotes) after exposure to very high temperatures. Biological Journal of the Linnean Society 93, 15-22.

NYLANDER, J.A.A. (2004). MrModeltest v2. Evolutionary Biology Centre, Uppsala University. Available online at http:// www.ebc.uu.se/systzoo/staff/nylander.html

RicCI, C. \& PAGANI, M. (1997). Desiccation of Panagrolaimus rigidus (Nematoda): survival, reproduction and the influence on the internal clock. Hydrobiologia 347, 1-13. 
Ronquist, F. \& HUELSENBECK, J.P. (2003). MRBAYES 3: Bayesian phylogenetic inference under mixed models. Bioinformatics 19, 1572-1574.

Shannon, A.J., Browne, J.A., Boyd, J., FitzPATRICK, D.A. \& BURNell, A.M. (2005). The anhydrobiotic potential and molecular phylogenetics of species and strains of Panagrolaimus (Nematoda, Panagrolaimidae). Journal of Experimental Biology 208, 2433-2445.

Thompson, J.D., Higgins, D.G. \& Gibson, T.J. (1994). CLUSTAL W: improving the sensitivity of progressive multiple sequence alignment through sequence weighting, positions-specific gap penalties and weight matrix choice. Nucleic Acid Research 22, 4673-4680.

Treonis, A.M. \& WALL, D.H. (2005). Soil nematodes and desiccation survival in the extreme arid environment of the Antarctic dry valleys. Integrative and Comparative Biology 45, 741-751.

Treonis, A.M., Wall, D.H. \& Virginia, R.A. (2000). The use of anhydrobiosis by soil nematodes in the Antarctic dry valleys. Functional Ecology 14, 460-467.

TUNNACLIFFE, A. \& WISE, M.J. (2007). The continuing conundrum of the LEA proteins. Naturwissenschaften 94, 791-812.

WHARTON, D.A. (1996). Water loss and morphological changes during desiccation of the anhydrobiotic nematode Ditylenchus dipsaci. Journal of Experimental Biology 199, 10851093.

WhARTON, D.A. (2002). Nematode survival strategies. In: Lee, D.L. (Ed.). The biology of nematodes. London, UK, Taylor \& Francis, pp. 389-411.
Wharton, D.A. (2003). The environmental physiology of Antarctic terrestrial nematodes: a review. Journal of Comparative Physiology B: Biochemical, Systemic, and Environmental Physiology 173, 621-628.

Wharton, D.A., BArrett, J. \& Perry, R.N. (1985). Water uptake and morphological changes during recovery from anabiosis in the plant parasitic nematode, Ditylenchus dipsaci. Journal of Zoology 206, 391-402.

Wharton, D.A., Petrone, L., Duncan, A. \& MCQuillan, A.J. (2008). A surface lipid may control the permeability slump associated with entry into anhydrobiosis in the plant parasitic nematode Ditylenchus dipsaci. Journal of Experimental Biology 211, 2901-2908.

Winston, P.W. \& BATES, D.H. (1960) Saturated solutions for the control of humidity in biological research. Ecology 41, 232-237.

WoMERSLEY, C. (1987). A reevaluation of strategies employed by nematode anhydrobiotes in relation to their natural enviroment. In: Veech, J.A. \& Dickson, D.W. (Eds). Vistas on nematology. Hyattsville, MD, USA, Society of Nematologists, Inc., pp. 165-173.

Womersley, C. \& Ching, C. (1989). Natural dehydration regimes as a prerequisite for the successful induction of anhydrobiosis in the nematode Rotylenchulus reniformis. Journal of Experimental Biology 143, 359-372. 